of some clever lemmas on derivations. As an application, the pfaffian of a 2-vector is defined, and an interesting proof is given of the equality of the determinants of an endomorphism and of its transposed endomorphism, both endomorphisms being considered (following another idea of Papy) as restrictions of a single endomorphism of the direct sum of the module $E$ and its dual.

Due to the usual time lag between research and teaching, multilinear algebra, although fundamental in modern mathematics, is still hardly taught (if taught at all) in most universities. It is to be hoped that many professors will avail themselves of the opportunity created by the publication of this little book, and let their students share the experience of the young Japanese mathematicians who first listened to these challenging lectures.

\title{
J. Dieudonné
}

Konstruktive Funktionentheorie. By I. P. Natanson. Trans. by K. Bögel. Berlin, Akademie-Verlag, 1955. $14+515$ pp. 36 DM.

The phrase "constructive theory of functions" was coined by S. Bernstein to describe the part of analysis that deals with the approximate representation of functions of a real variable by means of combinations of other functions. Thus it includes the theory of approximation, in various metrics, by polynomials and trigonometric polynomials; the theory of interpolation and approximate integration (formerly known inappropriately as "mechanical quadratures"); large portions of Fourier analysis and the theory of orthogonal functions; and related subjects like moment problems. All this may fairly accurately be thought of as the classical part of the subject. Although its fine structure (to borrow a term from atomic physics) is still undergoing investigation, the main results are at least 25 years old, often much older. There are also a number of topics that clearly belong to the subject but are of more recent development: approximation by translations of a function (and hence Wiener's Tauberian theorems); approximation by entire functions (developed by Kober and Bernstein within the last ten years); weighted polynomial approximation on infinite intervals (here the fundamental problem was finally solved by Pollard, and independently by Ahiezer and Bernstein, in 1953); closure and completeness theorems; extremal problems for polynomials, trigonometric polynomials, and more general classes of functions (currently enjoying a renaissance at the hands of Rogosinski and others); and the theory of special classes of functions that admit simple representations, such as absolutely mono- 
tonic functions and completely convex functions (which enjoyed a flurry of intensive activity a few years ago). The subject has been a favorite of Russian mathematicians from Chebyshev onwards, and especially of S. Bernstein and his students.

Ahiezer's Vorlesungen iber Approximationstheorie (translated into German in 1953 and reviewed in this Bulletin vol. 61, p. 369) dealt with parts of the subject at a rather advanced level and in monographic style, with emphasis on the modern theory. The present volume, translated from the Russian edition of 1951, is written as a textbook and covers the more elementary and classical portions in a more detailed style, especially in the first part. The author suggests that his book can be used as an introduction to the current literature or to such monographs as Ahiezer's. It is divided into three parts, dealing respectively with uniform approximation, $L^{2}$ approximation, and interpolation and approximate integration. The selection of modern topics seems to have been governed by their ability to fit into the classical framework. Thus Zygmund's smooth functions are included for their connection with best approximation, and there are some theorems of Marcinkiewicz and of Grünwald and Turán on interpolation. On the other hand, although Müntz's theorem on the completeness of $\left\{t^{\lambda_{n}}\right\}$ is included, there is no mention of the results of Clarkson and Erdös and of Schwartz on the span of such a set when it is not complete. The translator has added a rather spotty supplementary bibliography: it contains many papers published after the Russian edition of the book, but does not, for example, include anything by Mandelbrojt.

The contents are, in more detail, as follows. Part I: Approximation to continuous functions by polynomials and trigonometric polynomials. Best approximation of functions satisfying Lipschitz conditions, and converse theorems deducing properties of functions from the degree of approximation (the basic results are associated with the names of D. Jackson and S. Bernstein). Approximation by partial sums of Fourier series and by various transforms of these sums. The problem of best approximation to an analytic function of a real variable receives an original and completely real-variable treatment. Part II: Weighted $L^{2}$ approximation. Orthogonal polynomials and in particular the Legendre, Jacobi, Laguerre and Hermite systems. The Hausdorff and Hamburger moment problems. Part III: Interpolation polynomials of various kinds, general convergence and divergence theorems, modified interpolation processes; approximate integration formulas of various types. In part II the author mentions Nikolaev's theorem that no system of orthogonal polynomials can 
provide a convergent expansion for every continuous function. In an appendix he proves the following generalization by Lozinskii and Harshiladze: there exists no sequence $\left\{U_{n}\right\}$ of linear operators on $C$ such that $U_{n}$ takes every element of $C$ into a polynomial of degree at most $n$, and leaves such polynomials invariant, while $U_{n}(f) \rightarrow f$ for every $f$ in $C$.

The translation reads smoothly, with no relics of Russian mathematical style; it appears to be quite idiomatic, even to the extent of rendering "Buniakovskii's inequality" by "Cauchy's inequality" or "Schwarz's inequality" according to circumstances.

R. P. BOAS, JR.

\section{Brief Mention}

Allgemeine Theorie der algebraischen Zahlen. By Ph. Furtwängler. Reworked by $H$. Hasse and W. Jehne. Enzyklopädie der mathematischen Wissenschaften, vol. $\mathrm{I}_{2}$, no. 8, part II. 2d ed. Leipzig, Teubner, 1954. 50 pp. 6 DM.

This subject being surrounded in the Enzyklopädie by articles on Allgemeine Modul-Ring- und Idealtheorie, Bewertungstheorie, Theorie der abelschen Zahlkörper, and a section on analytic number theory, the authors faced a considerable problem in choice of material. In the reviewer's opinion they solved it admirably, allowing enough overlapping to make the various approaches to the subject clear. The first 40 pages are devoted to the arithmetic in integral domains of algebraic number fields as worked out by Kummer, Dedekind, and Kronecker, with an indication of the approach by valuation theory. The description of Kummer's approach, which is seldom mentioned but turns out to be surprisingly modern, is of interest. Main topics: ideals, ideal classes, different and discriminant, units. Except for a brief hint under "Axiomatische Begründung der Idealtheorie," there is no indication that practically all of this applies equally well to function fields of transcendence degree 1 . The last 10 pages are devoted to Artin's theory of the conductor and $L$-series, and analytic formulas for the class number.

\section{G. Whaples}

An introduction to stochastic processes with special reference to methods and applications. By M. S. Bartlett. Cambridge University Press, 1955. $312+14$ pp. $\$ 6.50$.

This book deals primarily with elementary heuristic applications to genetics, population growth, insurance risk, statistics, queuing 